Pathologe 2010 · 31:169-170

DOI 10.1007/s00292-009-1264-7

Online publiziert: 28. März 2010

๑) Springer-Verlag 2010

\author{
M. Vieth ${ }^{1} \cdot$ C. Kuhnen ${ }^{2}$ \\ ${ }^{1}$ Institut für Pathologie, Klinikum Bayreuth \\ ${ }^{2}$ Institut für Pathologie am Clemenshospital, Medical Center, Münster
}

\title{
Neues und Bewährtes aus der Magenpathologie
}

geleistet. Submuköse Tumoren werden, wenn günstig gelegen, mit Wedge-Resektionen entfernt. In vielen Krankenhäusern besteht eine ungeschriebene Übereinkunft, dass mesenchymale Tumoren über $2 \mathrm{~cm}$ Größe, oder wenn eindeutig maligne, eine Operationsindikation darstellen.

Herr Kuhnen beschreibt die Entität der atypischen lipomatösen Tumoren (gut differenziertes Liposarkom) im Magen in der vorliegenden Ausgabe von $\mathrm{Der} P \mathrm{~Pa}$ thologe im Detail anhand von zwei Fallbeispielen. Gerade diese im Gastrointestinaltrakt relativ seltenen Tumoren mit guter Prognose bereiten durch ihre Ähnlichkeit zu harmlosen Lipomen diagnostische Schwierigkeiten. Die vorliegende Arbeit gibt praktische Hinweise, wie diese Tumoren zu diagnostizieren und von Lipomen abzugrenzen sind.

Die Entität der erst seit 1990 bekannten gastral differenzierten Adenome des Magens („pyloric gland adenoma“) hat sich in Deutschland etabliert, nicht zuletzt durch entsprechende Schnittseminare verbreitet. Professor Borchard und Professor Watanabe haben zeitgleich und unabhängig voneinander 1990 diese Adenome beschrieben. Herr Kollege Kushima hat bei Herrn Borchard in Düsseldorf gearbeitet und ist so auf die Entität dieser gastral differenzierten Adenome gestoßen. Mittlerweile besitzt Herr Kushima am „National Cancer Center“ in Tokio die weltweite größte Sammlung dieser Adenome aus allen Lokalisationen im Gastrointestinaltrakt. In der vorliegenden Übersicht werden die diagnostischen Kriterien genau dargelegt. Zusätzlich haben wir das in Japan bestehende Konzept der gastralen Adenome beschrieben, letztendlich mit der Erkenntnis, dass zumindest diesbezüglich wenige Unterschiede in West und Ost bestehen.

Das Wissen um MALT-Lymphome und die Tatsache, dass durch eine Antibiotikatherapie ein maligner Tumor in die Remission gebracht werden kann, hat sehr viel zur Lebensqualität der Betroffenen beigetragen, die nun oftmals nicht operiert oder radio-chemotherapiert werden müssen. Gerade Herr Kollege Fischbach hat hier auf klinischer Seite sehr viel für die Patienten getan. Es lag also nahe, Herrn Fischbach und Herrn Eck aus Aschaffenburg für eine Publikation zu dem Thema Pathogenese, Diagnostik und Therapie der MALT-Lymphome zu gewinnen.

Frau Warich-Eitel - ebenfalls aus Aschaffenburg - konnte noch einen sehr seltenen Fall einer gastralen Leishmaniose für die aktuelle Ausgabe beisteuern. Die Leishmaniose wird durch Sandmücken im gesamten Mittelmeerraum übertragen und beispielsweise in Madrid jedes Jahr weit über 100-mal diagnostiziert. Gerade durch den zunehmenden Tourismus müssen wir Pathologen speziell bei verdächtigen Hautläsionen diesbezüglich sehr wachsam sein, wobei die intestinale Leishmaniose eher selten ist.

Nicht zuletzt durch die aktuelle TNMKlassifikation hat sich in den letzten Jahren bezüglich Diagnostik und therapeutischer Konsequenz bei neuroendokri-
Kollegin Wardelmann hat hier mit dem Aufbau des Referenzzentrums Großes 
nem Tumor einiges bewegt. Herrn Klöppels Verdienst ist es, dass neuroendokrine Tumoren in Deutschland einheitlich diagnostiziert werden - gerade bei Autoimmungastritis sind diese neuroendokrinen Tumoren ja oft wenig klinisch aggressiv. Seit Jahrzehnten fasst Herr Kollege Klöppel konsiliarische Zweitmeinungen mit großer Expertise gerade $\mathrm{zu}$ neuroendokrinen Tumoren ab und zeigt vor allem immer auch therapeutische Ratschläge auf. Zum Thema der neuroendokrinen Tumoren bot es sich also geradezu an, Herrn Kollegen Klöppel zu bitten, eine Übersichtsarbeit anzufertigen.

Ein ganz aktuelles Thema ist die Her2Expression bei Magenkarzinomen. Herr Rüschoff aus Kassel betreut als Pathologe seit langer Zeit die entsprechenden Studien. Die Her2/neu-Expression von Magenkarzinomen hat schon jetzt Einzug in die Routinediagnostik gefunden, wodurch Patienten mit Magenkarzinom noch eine zusätzliche Therapieoption geboten werden kann. Was bislang unklar war, ist die Graduierung der Her2/neu-Expression im Magen, da sich diese von der Mamma unterscheidet. Herrn Rüschoffs Verdienst ist es, in Deutschland eine einheitliche Graduierung entwickelt zu haben, die sich in der nächsten Zeit diagnostisch etablieren wird.

Herr Basten aus der Gruppe um Frau Kollegin Fisseler-Eckhoff aus Wiesbaden stellt eine elegante und kostengünstige Methode zur sicheren Identifikation von Lymphknoten an chirurgischen Präparaten dar, die natürlich nicht nur auf den Magen begrenzt ist, sondern an jeglichem Fettgewebe durchgeführt werden kann. Andere Methoden wie die Acetonfixation werden diskutiert und bezüglich Risiko und Nutzen verglichen. Gerade in Bezug auf qualitätsorientierte Probenbearbeitung bieten standardisierte Verfahren - zumal auch sehr klar dargestellt und effizient - natürlich immer Vorzüge.

Übrigens ist das vorliegende Themenheft das vorletzte, das der bisherige Schriftleiter von Der Pathologe, Professor Klaus-Michael Müller, in seiner Funktion als federführender Schriftleiter initiiert hat. An dieser Stelle möchten die Autoren Herrn Kollegen Müller noch einmal ausdrücklich für seine hervorragend gestalteten Themenhefte und sein Gespür für die
Vielfalt der Themen, die in Der Pathologe bislang erschienen sind, danken. Gerade die Qualität der Abbildungen ist ja zu einem Markenzeichen der Zeitschrift geworden.

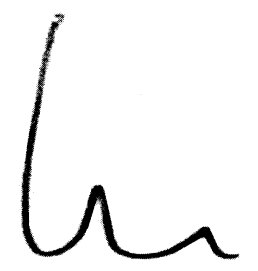

M. Vieth

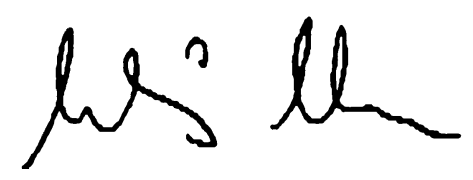

C. Kuhnen

\section{Korrespondenzadresse}

PD Dr. M. Vieth

Institut für Pathologie, Klinikum Bayreuth Preuschwitzerstr. 101, 95445 Bayreuth vieth.lkpathol@uni-bayreuth.de

\section{Gedeon, Andras \\ Fortschritte der Medizin durch Wissenschaft und Technik, 99 wegweisende Veröffent- lichungen aus fünf Jahrhun- derten}

Spektrum Akademischer Verlag

ISBN: 978-3-8274-2474-7, 59,95€

Wissenschaft und Medizin sind in ihrer Geschichte und Entwicklung eng verbunden. Außerdem haben technologischen Entdeckungen die Art und Weise grundlegend beeinflusst, in der Ärzte praktizieren, denn ohne die technischen Errungenschaften wären die vielen Fortschritte in der Medizin nicht möglich gewesen. Dank dieser Fortschritte erreichen wir heutzutage eine Lebenserwartung und Lebensqualität, wie sie frühere Generationen nie für möglich gehalten hätten.

Diesem Thema widmet sich das eben erschienene Buch „Fortschritte der Medizin durch Wissenschaft und Technik".

Der historische Bogen reicht von ersten mathematischen Körperproportionsanalysen Albrecht Dürers bis hin zur Entwicklung der Positronenemissionstomographie durch Michael Phelps. Eine Fundgrube des Wissens für alle, die an der Geschichte der Medizin, der Naturwissenschaften und der Technik interessiert sind. Der aufwändige Farbbildband ist eine Sammlung von 99 Essays über Meilenstein-Publikationen der letzten 500 Jahre aus einem weiten Feld wissenschaftlicher und technologischer Forschungsdisziplinen, die den Fortschritt der Medizin vorangebracht haben. Jeder Beitrag enthält eine Zusammenfassung der jeweiligen Publikation, eine kurze Biographie des Autors und eine Diskussion über den Einfluss der Entdeckung auf spätere Entwicklungen. Musterseiten aus dem ursprünglichen Artikel und Bilder aus der jeweiligen Zeit runden den Beitrag ab. Somit bekommt der Leser Original-Veröffentlichungen zu sehen (u.a. von Bernoulli, Doppler, Pasteur, Trendelenburg, Curie und Röntgen), die belegen, dass Fortschritt in der Medizin auf einer breiten internationalen und multidisziplinären Grundlage beruht.

Geschrieben wurde dieses Buch für Ärzte, Apotheker, Naturforscher, Wissenschaftler, Studierende der Human- und Veterinärmedizin sowie im Gesundheitswesen Tätige und für medizin-, medizintechnisch und wissenschaftshistorisch interessierte Sachbuchleser. 